\title{
Angular momentum spatial distribution symmetry breaking in Rb by an external magnetic field
}

\author{
Janis Alnis, Marcis Auzinsh* \\ Department of Physics, University of Latvia, 19 Rainis blvd., Riga LV-1586, \\ Latvia
}

(October 26, 2018)

Typeset using REVTEX

*Corresponding author, Fax +371-7820113, e-mail mauzins@latnet.lv 


\begin{abstract}
Excited state angular momentum alignment - orientation conversion for atoms with hyperfine structure in presence of an external magnetic field is investigated. Transversal orientation in these conditions is reported for the first time. This phenomenon occurs under Paschen Back conditions at intermediate magnetic field strength. Weak radiation from a linearly polarized diode laser is used to excite Rb atoms in a cell. The laser beam is polarized at an angle of $\pi / 4$ with respect to the external magnetic field direction. Ground state hyperfine levels of the $5 S_{1 / 2}$ state are resolved using laser-induced fluorescence spectroscopy under conditions for which all excited $5 P_{3 / 2}$ state hyperfine components are excited simultaneously. Circularly polarized fluorescence is observed to be emitted in the direction perpendicular to both to the direction of the magnetic field $\mathbf{B}$ and direction of the light polarization $\mathbf{E}$. The obtained circularity is shown to be in quantitative agreement with theoretical predictions.
\end{abstract}

\title{
I. INTRODUCTION
}

In the absence of external forces an ensemble of unpolarized atoms can only be aligned [1] by linearly polarized light. The fact that the atoms are only aligned implies that, although the magnetic sublevels of different $\left|m_{J}\right|$ are populated unequally, magnetic sublevels of $+m_{J}$ and $-m_{J}$ are equally populated. For this reason, atoms excited by linearly polarized laser radiation are not expected to produce circularly polarized fluorescence. In the presence of external forces, however, excitation by linear polarized light can produce an orientated population of atoms (with different $+m_{J}$ and $-m_{J}$ populations.) This effect, called alignment - orientation conversion, was predicted and experimentally observed in the late sixties in the anisotropic collisions of initially aligned atoms [2 [5]. Later an electric field was also shown to induce alignment - orientation conversion [6]. Electric-field-induced alignment - 
orientation conversion has since been studied in great detail [7].

Contrary to the case of an electric field, linear perturbation by a magnetic field is not able to orient an initially aligned angular momentum distribution. This inability to induce alignment - orientation conversion is a result of the reflection symmetry of axial vector fields. This symmetry can be broken if, in addition to the linear Zeeman effect, there exists nonlinear dependencies of the magnetic sublevel energies on the field intensity and the magnetic quantum numbers $m_{J}$. Such nonlinear perturbations can have a variety of causes including predissociation 8 -11] and hyperfine interaction. Alignment - orientation conversion as a result of hyperfine interaction in a magnetic field in context of nuclear spin $I=1 / 2$ was studied by J. Lehmann for the case of optically pumped cadmium in a magnetic field [12,13]. W. Baylis described the same effect in sodium [14]. The first experiment to detect directly a net circular polarization of fluorescence from an initially aligned excited state in an external magnetic field was reported by M. Krainska - Miszczak [15]. In this work the optical pumping of ${ }^{85} \mathrm{Rb}$ by a $\pi$-polarized $\mathrm{D}_{2}$ line was studied. This effect was also examined by X. Han and G. Schinn in sodium atoms [16]. They describe this alignment - orientation conversion process as resulting from hyperfine- $F$-level mixing in an external magnetic field and the interference of different excitation - decay pathways in such mixed levels.

In all above cases, a joint action of the magnetic field and hyperfine interaction creates different population of magnetic sublevels $+m_{F}$ and $-m_{F}$ of hyperfine levels $F$. This means that longitudinal orientation of atoms along the direction of an external magnetic field is created. Recently it was predicted that joint action of a magnetic field and hyperfine interaction from an initially aligned ensemble would create transverse orientation of angular momentum of atoms or molecules [17]. Transverse orientation implies orientation in a direction perpendicular to the external magnetic field B. In this particular case magnetic sublevels $+m_{F}$ and $-m_{F}$ are equally populated, but orientation is a result of coherence between pairs of wave functions of magnetic sublevels $m_{F}$ with $\Delta m_{F}=1$. Creation of transverse orientation is achieved if the excitation light polarization vector is neither parallel nor perpendicular 
to the external magnetic field direction with the largest effect occurring for the case of a light polarization - magnetic field angle of $\pi / 4$. In a previous paper [17], parameters of the NaK molecule were used for numerical simulations of orientation and fluorescence circularity signals. We found that transverse orientation only occurred when the rotational angular momentum $J$ is small enough to be comparable with the nuclear spin $I$. For levels with larger angular momentum quantum number, the magnitude of created orientation was found to decrease rapidly.

Previously transverse alignment - orientation conversion was studied in detail for the

case of an external electric field [7]. In this case the conversion occurs with or without hyperfine interaction. In this paper we report the first experimental observation to our knowledge of alignment - orientation conversion that creates net transverse orientation of atoms with hyperfine structure in an external magnetic field. As we will show, this effect is interesting not only as a new way to create orientated atoms, but also can be used to increase the accuracy with which constants related to the hyperfine interaction can be determined.

\section{THEORETICAL DESCRIPTION}

The general scheme how transverse orientation of angular momentum is created from an aligned ensemble of atoms is the following: Initial alignment, for example by absorption of a linearly polarized light, is created at some non-zero acute angle with respect to the direction of an external-field (in this case a B-field.) The optimum angle is $\pi / 4$, but the effect will take place at any angle that differs form 0 and $\pi / 2$. The perturbing field together with the hyperfine interaction causes unequally spaced magnetic sublevel splittings. Under these conditions, angular momenta orientation at the direction perpendicular to the direction of the external field is created [22]. A semiclassical interpretation of this effect in terms of angular momentum precession in an external field can be found in a previous publication [7]. In this vectorial model, alignment - orientation conversion is the result of a different precession rate for different orientations of angular momentum with respect to the external 
field. In what follows we explain this transverse-orientation in terms of a accurate quantum mechanical model.

In the present study we exploit laser excitation of pure isotopes of Rb atoms from their ground state $5 S_{1 / 2}$ to the first excited state $5 P_{3 / 2}$ (resonance $\mathrm{D}_{2}$ line) (see inset Figure 5.) The two most common naturally occurring isotopes of Rubidium are ${ }^{85} \mathrm{Rb}(72.15 \%$, nuclear spin $I=5 / 2)$ and ${ }^{87} \mathrm{Rb}(27.85 \%$, nuclear spin $I=3 / 2$.) As a result of hyperfine interactions, the ground-state level of ${ }^{85} \mathrm{Rb}$ is split into components with total angular momentum quantum numbers $F_{i}=2$ and $F_{i}=3$ and the ground-state level of ${ }^{87} \mathrm{Rb}$ is split into components with total angular momentum quantum numbers $F_{i}=1$ and 2 . The ground-state-level splittings for ${ }^{85} \mathrm{Rb}$ and ${ }^{87} \mathrm{Rb}$ are approximately $3 \mathrm{GHz}$ and $6 \mathrm{GHz}$ respectively. In contrast, the four excited state hyperfine components are separated by only several hundred $\mathrm{MHz}$ (see Figures [1 and 3).

Excited-state-hyperfine structure in absorption is not resolved due to Doppler broadening and laser-frequency jittering. To make an accurate signal modeling assuming broad line excitation, laser frequency is modulated by a few hundred $\mathrm{MHz}$ superimposing a $10 \mathrm{kHz}$ sine wave on laser current. This allows accurate modeling to be done assuming that the excitation radiation is broad enough to excite all hyperfine components of the excited state without frequency selection, yet narrow enough to completely resolve the two ground-state components.

Magnetic field caused mixing takes place between sublevels with different total angular momentum $F_{e}$, but with identical magnetic quantum numbers $m_{F}$. Only levels of identical $m_{F}$ mix because, as far as the magnetic field possesses axial symmetry, the magnetic quantum number $m_{F}$ remains a good quantum number. However, levels of different $F_{e}$ mix because an intermediate strength magnetic field partially decouples the electronic angular momentum $J_{e}$ and nuclear spin $I$. As a consequence $F_{e}$ ceases to be a good quantum number. The fact the $m_{F}$ remains a good quantum number whereas $F_{e}$ does not is important to the interpretation of the data.

A convenient way to describe excited state atoms is by means of a quantum density 
matrix ${ }^{k l} f_{m m^{\prime}}[18]$. Upper indices characterize atomic states in a magnetic field. In the weak field limit these states correspond to hyperfine levels $F_{e}$. Lower indices characterize magnetic quantum numbers. We consider an atom possessing the hyperfine structure which is placed in an external magnetic field. We further assume that this atom absorbs laser light polarized in the direction characterized by light electric field vector $\mathbf{E}_{\text {exc }}$. In this situation the density matrix that characterizes coherence between magnetic sublevels with quantum numbers $m$ and $m^{\prime}$ is given as 21]

$$
{ }^{k l} f_{m m^{\prime}}=\frac{\widetilde{\Gamma}_{p}}{\Gamma+i^{k l} \Delta \omega_{m m^{\prime}}} \sum_{j \mu}\left\langle\gamma_{k} m\left|\widehat{\mathbf{E}}_{e x c}^{*} \cdot \widehat{\mathbf{D}}\right| \eta_{j} \mu\right\rangle\left\langle\gamma_{l} m^{\prime}\left|\widehat{\mathbf{E}}_{e x c}^{*} \cdot \widehat{\mathbf{D}}\right| \eta_{j} \mu\right\rangle^{*}
$$

Here $\widetilde{\Gamma}_{p}$ is a reduced absorption rate, $\Gamma$ is the excited state relaxation rate and ${ }^{k l} \Delta \omega_{m m^{\prime}}=$ $\left({ }^{\gamma_{k}} E_{m}-\gamma_{l} E_{m^{\prime}}\right) / \hbar$ is the energy splitting of magnetic sublevels $m$ and $m^{\prime}$ belonging to the excited state levels $k$ and $l$. Magnetic quantum numbers of the ground state level $\eta_{j}$ are denoted by $\mu$ and magnetic quantum numbers of the excited state level $\gamma_{k}$ by $m$ and $m^{\prime}$.

In an external magnetic field, ground- and excited- state levels $\eta_{j}$ and $\gamma_{k}$ are not characterized by a total angular momentum quantum numbers $F_{i}$ and $F_{e}$, but are instead mixtures of these states:

$$
\left|\gamma_{k} m\right\rangle=\sum_{F_{e}=J_{e}-I}^{F_{e}=J_{e}+I} C_{k F_{e}}^{(e)}\left|F_{e}, m\right\rangle, \quad\left|\eta_{j} \mu\right\rangle=\sum_{F_{i}=J_{i}-I}^{F_{i}=J_{i}+I} C_{j F_{i}}^{(i)}\left|F_{i}, \mu\right\rangle .
$$

The wave-function-expansion coefficients $C_{k F_{e}}^{(e)}, C_{j F_{i}}^{(i)}$ represent the mixing of field free hyperfine state wave functions by the magnetic field. These expansion coefficients along with the magnetic sublevel energy splittings ${ }^{k l} \Delta \omega_{m m^{\prime}}$ can be obtained by a standard procedure of diagonalization of a Hamilton matrix that contains both the diagonal hyperfine elements and the off-diagonal magnetic field interaction elements (see for example [17].)

There are several methods how to tell whether or not a particular atomic state described by a density matrix (四) possesses orientation. One possibility is to expand this matrix over the irreducible tensorial operators. Then those expansion coefficients can directly be attributed to the alignment and orientation of the atomic ensemble [18 20]. Alternatively, 
one may calculate directly the fluorescence circularity rate in spontaneous transitions from a particular excited state of an atom:

$$
C=\frac{I\left(\mathbf{E}_{\text {right }}\right)-I\left(\mathbf{E}_{\text {left }}\right)}{I\left(\mathbf{E}_{\text {right }}\right)+I\left(\mathbf{E}_{\text {left }}\right)}
$$

Observed circularity of the fluorescence in a specific direction can differ from zero only for the case that the ensemble of atoms possesses overall orientation in this direction 18 . $I\left(\mathbf{E}_{\text {right }}\right)$ and $I\left(\mathbf{E}_{l e f t}\right)$ are intensities of two fluorescence components with opposite circularity. We choose to calculate this expected circularity rate because it is the experimental measure used to register the appearance of orientation in an ensemble of atoms (see for example [22]).

We consider the case that spontaneous emission is detected without hyperfine-state resolution. The intensity of the fluorescence with definite polarization characterized by a vector $\mathbf{E}_{f}$ in a spontaneous transition from an excited state $J_{e}$ characterized by a set $\gamma_{k}$ of levels in an external field to the ground state $J_{f}$ characterized by a set $\eta_{j}$ of levels can be calculated according to a previous work [21] as

$$
I\left(\mathbf{E}_{f}\right)=I_{0} \sum_{m m^{\prime} \mu} \sum_{k l j}\left\langle\gamma_{k} m\left|\widehat{\mathbf{E}}_{f}^{*} \cdot \widehat{\mathbf{D}}\right| \eta_{j} \mu\right\rangle\left\langle\gamma_{l} m^{\prime}\left|\widehat{\mathbf{E}}_{f}^{*} \cdot \widehat{\mathbf{D}}\right| \eta_{j} \mu\right\rangle^{* k l} f_{m m^{\prime}}
$$

To find the circularity rate $C$, one needs to not only determine the matrix elements appearing in (1) and (4), but also the hyperfine level splitting and magnetic sublevel mixing coefficients. In Figure 1 the hyperfine energy level splitting of the first excited state $5 P_{3 / 2}$ for ${ }^{85} \mathrm{Rb}$ is presented. In these calculations the following published 23 hyperfine splitting constants and magnetic moment for the rubidium atom in its first excited state are used: $a=25.009$ $\mathrm{MHz}, b=25.83 \mathrm{MHz}, g_{J}=-1.3362, g_{I}=0.000293$.

In Figure 11 level crossing positions for magnetic sublevels with $\Delta m_{F_{e}}=2$ are indicated by circles and crossings with $\Delta m_{F_{e}}=1$ by squares. At values of magnetic field strength for which coherently excited magnetic sublevels undergo a level crossing, ${ }^{k l} \Delta \omega_{m m^{\prime}}=0$ the prefactor appearing in Equation (四) becomes large. This leads to resonance behavior of the observed signal. For case of excitation with linearly polarized light, the intensity of the resonance depends upon the angle between polarization direction of the laser light 
and external magnetic field direction. If the angle between these directions is 0 , different magnetic sublevels are differently populated but no coherence is created in the ensemble. If the angle is $\pi / 2$ coherence is created between magnetic sublevels with $\Delta m_{F_{e}}=2$. If the angle differs form 0 and $\pi / 2$ then magnetic sublevels with $\Delta m_{F_{e}}=1$ and 2 [22] are excited coherently. This $\Delta m_{F_{e}}=1$ coherence is required for transverse orientation.

We now consider the fluorescence circularity enhancement due to $\Delta m_{F_{e}}=1$ level crossing for the case that the linear polarization and external field meet at an angle of $\pi / 4$ (inset Figure 2.) The circularity $C$ is calculated assuming an excited state relaxation rate 24] $\Gamma=3.8 \times 10^{7} \mathrm{~s}^{-1}$ and observation along an axis normal to the plain containing the external field $\mathbf{B}$ and the polarization vector $\mathbf{E}_{\text {exc }}$. The smooth lines of Figure 2 give the expected signals for both resolved absorption lines. Both signals are maximum at an approximate magnetic field strength of $10 \mathrm{G}$. For both absorption lines we calculate a total fluorescence circularity with unresolved hyperfine components in a transition back to the ground state $5 S_{1 / 2}$. The resonance peak is more pronounced for the $F_{i}=2 \longrightarrow F_{e}$ absorption transition than for the $F_{i}=3 \longrightarrow F_{e}$ transition.

Because a $10 \mathrm{G}$ field is weak enough not to cause substantial hyperfine level mixing (i.e., the magnetic sublevel splitting in the magnetic field still is small in comparison with hyperfine splitting, ) the increase in orientation for $F_{i}=2$ absorption can be understood using the relative transition probability $W_{F_{i}} \rightarrow F_{e}$ given by Sobelman [20]:

$$
W_{F_{i} \longrightarrow F_{e}}=\left(2 F_{i}+1\right)\left(2 F_{e}+1\right)\left(2 J_{i}+1\right)\left(2 J_{e}+1\right)\left\{\begin{array}{ccc}
J_{i} & F_{i} & I \\
F_{e} & J_{e} & 1
\end{array}\right\}\left\{\begin{array}{ccc}
L_{i} & J_{i} & S \\
J_{e} & L_{e} & 1
\end{array}\right\}^{2} .
$$

Here $J_{i}, J_{e}$ and $L_{i}, L_{e}$ are quantum numbers of total and orbital electronic angular momentum of the initial and final atomic state and $S$ is the electronic spin of the atomic state. Quantities in curled brackets are $6-j$ symbols. This expression predicts that the $F_{i}=2 \longrightarrow F_{e}=2$ absorption contributes $39 \%$ of the total allowed ( $\left.\Delta F=0, \pm 1\right)$ absorption from $F_{i}=2$. In contrast, the $F_{i}=3 \longrightarrow F_{e}=2$ absorption contributes only $8 \%$ of the total allowed $(\Delta F=0, \pm 1)$ absorption from $F_{i}=3$. At the same time the $F_{e}=2$ state is 
the state for which the magnetic sublevels undergo a level crossing in the vicinity of a 10 G magnetic field. Thus the absorption from the $F_{i}=2$ state leads to a greater degree of transverse orientation.

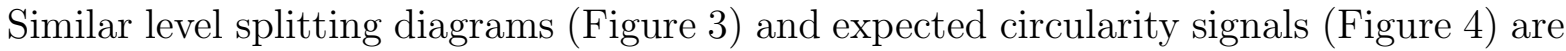
calculated also for rubidium isotope ${ }^{87} \mathrm{Rb}$. In this case the following atomic constants are used: $I=3 / 2, a=84.845 \mathrm{MHz}, b=12.52 \mathrm{MHz}, g_{J}=-1.3362, g_{I}=-0.000995$ [23].

\section{EXPERIMENTAL}

In our experiment we use isotopically enriched rubidium (99\% of $\left.{ }^{85} \mathrm{Rb}\right)$ contained in a glass cell at room temperature to keep atomic vapor concentration low and avoid reabsorption. The $5 s{ }^{2} S_{1 / 2}$ to $5 p^{2} P_{3 / 2}$ transition at $780.2 \mathrm{~nm}$ is excited using a temperature- and current- stabilized single-mode diode laser (Sony SLD114VS). Absorption signal is measured using a photodiode. As the laser frequency is swept using a ramped current drive, two absorption peaks with half-width of about $600 \mathrm{MHz}$ separated by $\sim 3 \mathrm{GHz}$ appear due to the ${ }^{85} \mathrm{Rb}$ ground state hyperfine structure. The excited-state hyperfine structure is not resolved under the Doppler profile and introduced laser-frequency jittering. The laser line width without jittering is about $60 \mathrm{MHz}$. To avoid optical pumping and other nonlinear effects, neutral density filters are used to reduce the laser intensity until absorption lines at $60 \mathrm{G}$ broaden by less than $10 \%$.

During the level crossing and circularity measurements, the laser wavelength is stabilized on one of the two absorption peaks. Fluorescence is monitored on an axis normal to the electric vector $\mathbf{E}_{\text {exc }}$ and external magnetic field $\mathbf{B}$. A two-lens system is used to image the fluorescence on a photodetector containing a $3 \times 3 \mathrm{~mm}$ photodiode (Hamamatsu S1223$01)$ and a transimpedance amplifier. A rotating $(f=240 \mathrm{~Hz})$ sheet polarizer is inserted between the lenses. The photodetector signal is fed to a lock-in amplifier (Femto LIA-MV150) that measures the intensity difference of two orthogonal linearly polarized fluorescence components. A magnetic field of up to $65 \mathrm{G}$ is produced by passing current through a pair 
of Helmholz coils $20 \mathrm{~cm}$ in diameter. The uncertainty of the magnetic field is estimated to $\pm 0.3 \mathrm{G}$. The sweep time is $5 \mathrm{~s}$ and 256 sweeps are averaged on an IBM compatible computer with a National Instruments data acquisition card. A lock-in time constant of $10 \mathrm{~ms}$ is used. Several adjustments are made to record symmetrical level crossing signals while sweeping the magnetic field in opposite directions. First, a linear polarizer is placed in a laser beam before the rubidium cell to fine adjust the laser polarization. Second, the lock-in phase is adjusted and, third, the Earth magnetic field components are compensated with additional Helmholz coils.

To detect circularly polarized light the gain electronics are first adjusted so that the linear polarization signals are symmetrical in opposite magnetic field directions. A $\lambda / 4$ wave plate is then placed before the polarizer so that right- and left- handed circularly polarized light components are converted to opposite polarizations. It is checked that circularity signal at $B=0$ is zero. During the circularity measurements the magnetic field is swept alternatively in one and another direction and both traces are averaged. The experimentally recorded signal actually is $I\left(\mathbf{E}_{\text {right }}\right)-I\left(\mathbf{E}_{\text {left }}\right)$ and not the ratio $\left(I\left(\mathbf{E}_{\text {right }}\right)-I\left(\mathbf{E}_{\text {left }}\right)\right) /\left(I\left(\mathbf{E}_{\text {right }}\right)+\right.$ $\left.I\left(\mathbf{E}_{l e f t}\right)\right)$. Numerical simulations reveal that these two signals have almost the same shapes, the relative difference is less than $3 \%$. Experimentally recorded signals are scaled vertically to fit the calculated ones.

Figures 8 and 4 compare experimentally obtained circularity to the theoretical ones. After the scaling factor to the experimental signal is applied (no other adjustable parameters are used) an excellent agreement between theoretical predictions and experimental signals can be observed. For both isotopes circularity signals with amplitude of several percents are measured.

In case of the measurements with ${ }^{87} \mathrm{Rb}$ another cell was used, that contained isotopically enriched ${ }^{87} \mathrm{Rb}(99 \%)$. For this isotope the signal starting from $F_{i}=1$ exhibits stronger resonance circularity than the one starting from the $F_{i}=2$ ground state. The reason for this is the same as already discussed in a Section $\left[\mathrm{I}\right.$ for the ${ }^{85} \mathrm{Rb}$ isotope. Only in this case the excited state hyperfine component $F_{e}=1$ undergoes level crossings with $\Delta m_{F_{e}}= \pm 1$. 
This resonance intensity ratio for two measured signals reflects the general situation that transitions with $\Delta F=0$ are more intense than transitions with $\Delta F= \pm 1$.

\section{COMPARISON OF CIRCULARITY MEASUREMENTS TO OTHER LEVEL-CROSSING MEASUREMENTS}

In previous studies, atoms are excited by a linearly polarized light with $\mathbf{E}_{e x c}$ vector perpendicular to an external magnetic field. The fluorescence emitted along the magnetic field is then detected. Fluorescence linear polarization as a function of magnetic field is

measured. Here we repeat this experiment for the case of ${ }^{85} \mathrm{Rb}$ (see inset of Figure 5.) Two signals are numerically simulated and experimentally recorded, the first one when absorption occurs on the transitions $\left(F_{i}=2 \rightarrow F_{e}\right)$ and a second one for a $\left(F_{i}=3 \rightarrow F_{e}\right)$ absorption transition. In both cases the conditions are maintained so that the excited state hyperfine levels are not resolved. For the first absorption transition in the absence of the magnetic field electric dipole transitions are allowed only to the levels $F_{e}=1,2$, and 3 . For the second absorption transition in absence of the external field hyperfine components with $F_{e}=2,3$, and 4 can be excited.

In presence of the magnetic field selection rules change substantially. As it was mentioned before, $F_{e}$ is no longer a good quantum number. Each hyperfine level in the presence of external field is mixed together with others. As far as $m_{F_{e}}$ remains a good quantum number in the presence of the external field, only components with the same $m_{F_{e}}$ are mixed. This implies that for the present example of ${ }^{85} \mathrm{Rb}$, magnetic sublevels with $m_{F_{e}}=4$ and -4 at any field value are unmixed because only $F_{e}=4$ contains such sublevels and there is no counterpart for these states to be mixed with. In case of $m_{F_{e}}=3$ and -3 only two magnetic sublevels originating from $F_{e}=3$ and 4 are mixed together, etc. This means that magnetic sublevels $m_{F_{e}}=0, \pm 1$ in external field are composed from $F_{e}=1,2,3,4$ components, $m_{F_{e}}= \pm 2$, from $F_{e}=2,3,4$ components $m_{F_{e}}= \pm 3$, from $F_{e}=3,4$ components but $m_{F_{e}}= \pm 4$, contain only one component $F_{e}=4$. 
In Figure 1 we can see several $\Delta m_{F_{e}}=2$ magnetic sublevel crossings. The first crossing takes place at zero magnetic field when all magnetic sublevels belonging to the same hyperfine level have the same energy. This crossing is the zero field level crossing. Because all magnetic sublevels belonging to the same hyperfine state cross at zero field, the zero field level crossing leads to the largest resonance amplitude. Then subsequent crossings take place at approximately 2.4, 4.2, 8.2, 24, 44, 52 and $74 \mathrm{G}$ magnetic field strength. A resonant peak occurs on both linear polarization signals for almost every one of these level crossings, although with differing amplitudes (see Figure 5.) There is one exception. The strong resonance peak at $52 \mathrm{G}$ that is present in $F_{i}=3 \rightarrow F_{e}$ signal is missing in $F_{i}=2 \rightarrow F_{e}$ signal. This seeming inconsistency can be easily explained. This resonance appears when the magnetic sublevels $m_{F_{e}=4}=-4$ and $m_{F_{e}=3}=-2$ are crossing. But as it was mentioned due to dipole transition selection rules the $m_{F_{e}=4}=-4$ level can not be excited from $F_{i}=2$ and this restriction can not be removed by external field because $m_{F_{e}=4}=-4$ remains unmixed at any field strength.

In the same Figure 5 along with the theoretically simulated signal the experimentally registered signal is depicted as well. The only adjustable parameter in this comparison is a scaling factor for the overall intensity of the experimentally detected signal. The observed signals agrees very well with level crossing signal registered by several groups before us [24,25]. However, in these previous studies the first derivative from the intensity was measured and so we were able to compare only the exact positions of resonances. These coincide perfectly. In our case we are able to calculate not only the positions of resonances, but also the shape, width and relative amplitudes of the resonance peaks.

\section{CONCLUSIONS}

In this study we report for the first time the appearance of transverse orientation in atoms with hyperfine structure after excitation by linearly polarized light in the presence of an external magnetic field. We have also presented a theory that is in quantitative agreement 
with our data. The use of transverse alignment-orientation as a probe of level crossings is compared to previous measurements The case of measured circularity has an advantage over the more conventional measurement of the degree of linear polarization: For the case of alignment - orientation conversion, there is no signal in the absence of the external field. This implies that we do not have the first trivial resonance position at zero field value which is always present in traditional geometry (Hanle effect [26].) This allows measurements of first level crossing positions that are very close to the zero field resonance. In traditional methods these resonances are hidden under the zero field peak. For example, from the inset of Figure 1 we can see that there must exist several resonances of $\Delta m_{F_{e}}=2$ crossings around 3 and $6 \mathrm{G}$. However these resonances are hidden in a traditional level crossing signal and can not be observed (see Figure 5.) At the same time $\Delta m_{F_{e}}=1$ crossings that appear even at smaller field values 2,4 and $8 \mathrm{G}$ in alignment - orientation conversion signals, although not fully resolved are clearly visible. The possibility to detect these resonances can improve the precision of atomic hyperfine splitting constants.

\section{ACKNOWLEDGMENTS}

This work could not be completed without valuable comments and advice of Dr. Habil. Phys. Maris Tamanis from Institute of Atomic Physics and Spectroscopy, University of Latvia and continuous support of Prof. Sune Svanberg from Department of Physics at

Lund Institute of Technology. We are thankful to Prof. Neil Shafer-Ray from University of Oklahoma for careful reading of the manuscript and valuable comments. Financial support from Swedish Institute Visby program is greatly acknowledged. 


\section{REFERENCES}

[1] In this work the terms alignment, orientation, and polarization take on their technical meaning. Alignment refers to a distribution of angular momentum direction with respect to an axis, but not a preferred direction. Orientation refers to a distribution of angular momentum direction that favors a given direction.

[2] M. Lombardi, C. R. Acad. Sci., Ser. B, 265B, 191 (1967)

[3] V.N. Rebane, Opt. Spectrosc. USSR, 51, 163 (1968)

[4] M. Lombardi, in Beam Foil Spectroscopy, edited by I.A. Sellin and D.J. Pegg, vol. 2, (Plenum Press, New York, 1976), p. 731

[5] T. Manabe, T. Yabuzaki, T. Ogawa, Phys. Rev. Lett., 46, 637 (1981)

[6] M. Lombardi, J. Phys. (Paris), 30, 631 (1969)

[7] M. Auzinsh, Can. J. Phys. 75, 853 (1997); M. Auzinsh, R. Ferber, Phys. Rev. Lett. 69, 3463 (1992); M. Auzinsh, R. Ferber, and A.V. Stolyarov. J. Chem. Phys. 101, 5559 (1994).

[8] J. Vigue, M. Broyer, J.-C. Lehmann, J. Phys. B, 7, L158, (1974)

[9] J. Vigue, M. Broyer, J.-C. Lehmann, J. Chem. Phys. 62, 4941 (1975)

[10] I. Klintsare, M. Tamanis, A.V. Stolyarov, M. Auzinsh and R. Ferber, J. Chem. Phys. 99, $5748(1993)$

[11] M. Auzinsh, A.V. Stolyarov, M. Tamanis, R. Ferber, J. Chem. Phys., 105, 37 (1996)

[12] J.C. Lehmann, Journal de Physique, 25, 809 (1964)

[13] J.C. Lehmann, Phys. Rev. 178, 153 (1969)

[14] W.E. Baylis, Phys. Lett., 26A, 414 (1968)

[15] M. Krainska - Miszczak, J. Phys. B: Atom. Molec. Phys., 12, 555 (1979) 
[16] X.L. Han, G.W. Schinn, Phys. Rev. A, 43, 266 (1991)

[17] J. Alnis, M. Auzinsh, European Physical Journal D, 11, 91 (2000)

[18] M. Auzinsh, R. Ferber, Optical Polarization of Molecules, (Cambridge University Press, Cambridge UK, 1995)

[19] D.A. Varshalovich, A.N. Moskalev, V.K. Khersonskii, Quantum Theory of Angular Momentum, (World Scientific, Singapore, 1988).

[20] I.I. Sobelman, Atomic Spectra and Radiative Transitions, (Springer-Verlag, Berlin, 1979)

[21] M. Tamanis, M. Auzinsh, I. Klincare, O. Nikolayeva, A. V. Stolyarov, R. Ferber, J. Chem. Phys., 106, 2195 (1997)

[22] M. Auzinsh, R. Ferber, J. Chem. Phys. 99, 5742 (1993)

[23] E. Arimondo, M. Inguscio, P. Violino, Rev. Mod. Phys, 49, 31 (1977)

[24] G. Belin, S. Svanberg, Physica Scripta 4, 269 (1971)

[25] R.W. Schmieder, A. Lurio, W. Happer and A. Khadjavi, Phys. Rev. A2, 1217 (1970)

[26] G. Moruzzi, F. Strumia, Hanle Effect and Level-Crossing Spectroscopy, (Plenum Press, New York, London, 1991). 


\section{FIGURES}

FIG. 1. Hyperfine structure energy-level diagram of ${ }^{85} \mathrm{Rb} 5 p^{2} P_{3 / 2}$ in an external magnetic field. Symbols $\square$ indicate $\Delta m_{F_{e}}=1$ level crossings, $\bigcirc-\Delta m_{F_{e}}=2$ level crossings.

FIG. 2. Numerically simulated (smooth line) and experimentally measured (signal with noise) level crossing signals in fluorescence circularity for ${ }^{85} \mathrm{Rb}$ in conditions of alignment - orientation conversion and production of transversal orientation.

FIG. 3. Hyperfine structure energy-level diagram of ${ }^{87} \mathrm{Rb} 5 p^{2} P_{3 / 2}$ state in an external magnetic field. Symbols $\square$ indicate $\Delta m_{F_{e}}=1$ level crossings, and $\bigcirc-\Delta m_{F_{e}}=2$ level crossings.

FIG. 4. Numerically simulated (smooth line) and experimentally measured (signal with noise) level crossing signals in fluorescence circularity for ${ }^{87} \mathrm{Rb}$ in conditions of alignment - orientation conversion and production of transversal orientation.

FIG. 5. Numerically simulated (smooth line) and experimentally measured (signal with noise) level crossing signals in linearly polarized fluorescence for ${ }^{85} \mathrm{Rb}$. 


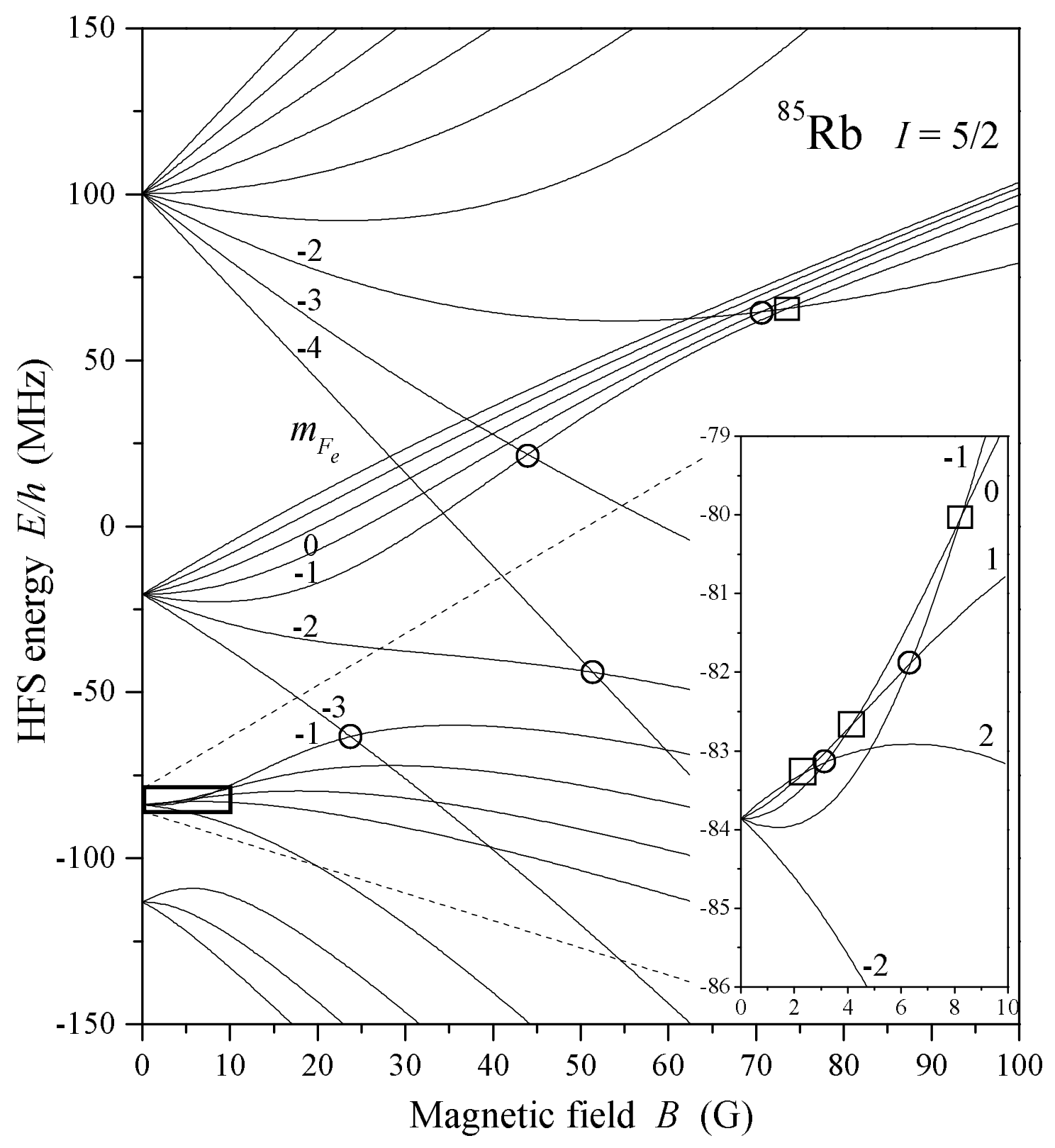

Figure 1

J.Alnis and M.Auzinsh 


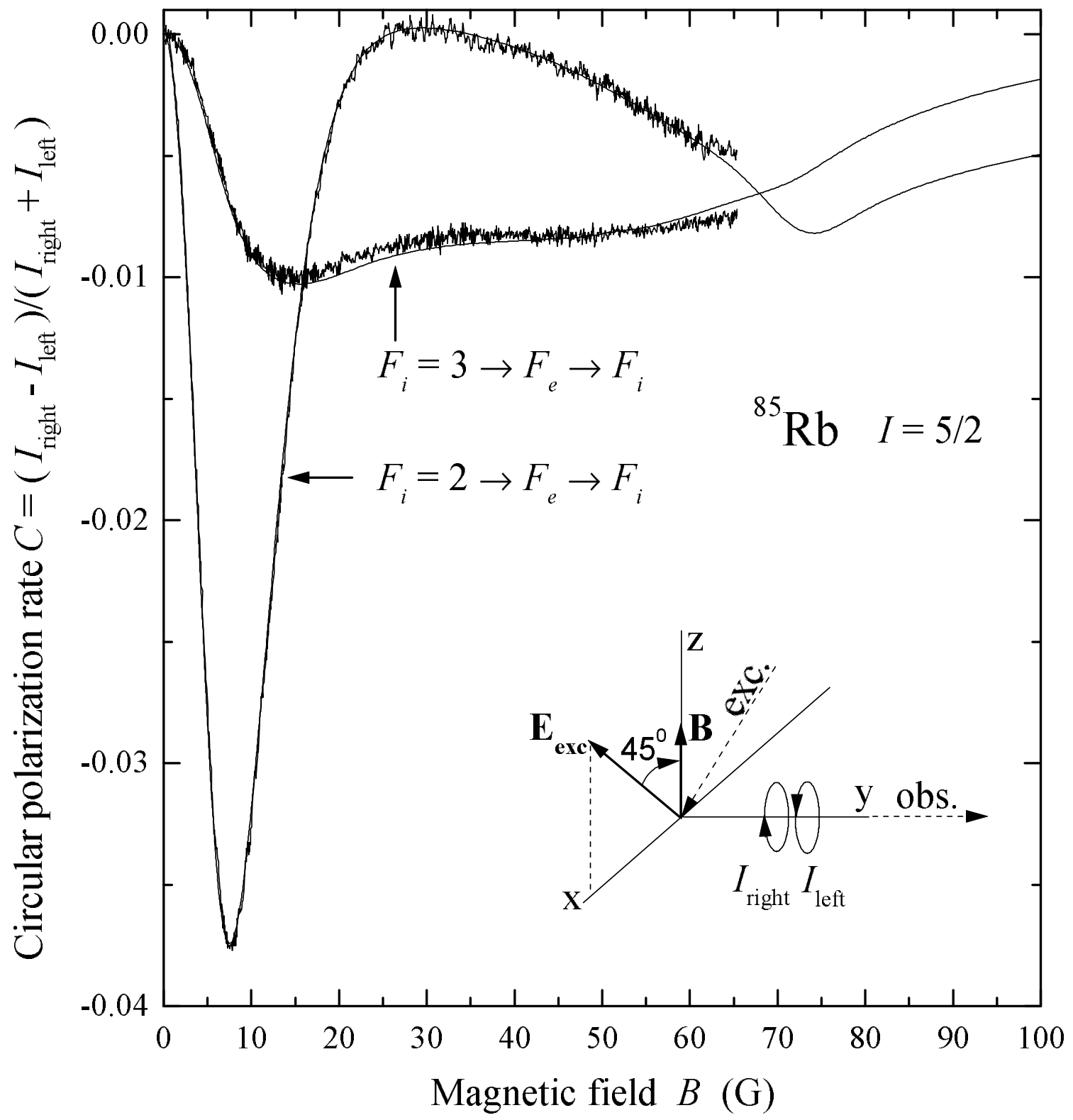

Figure 2

J.Alnis and M.Auzinsh 


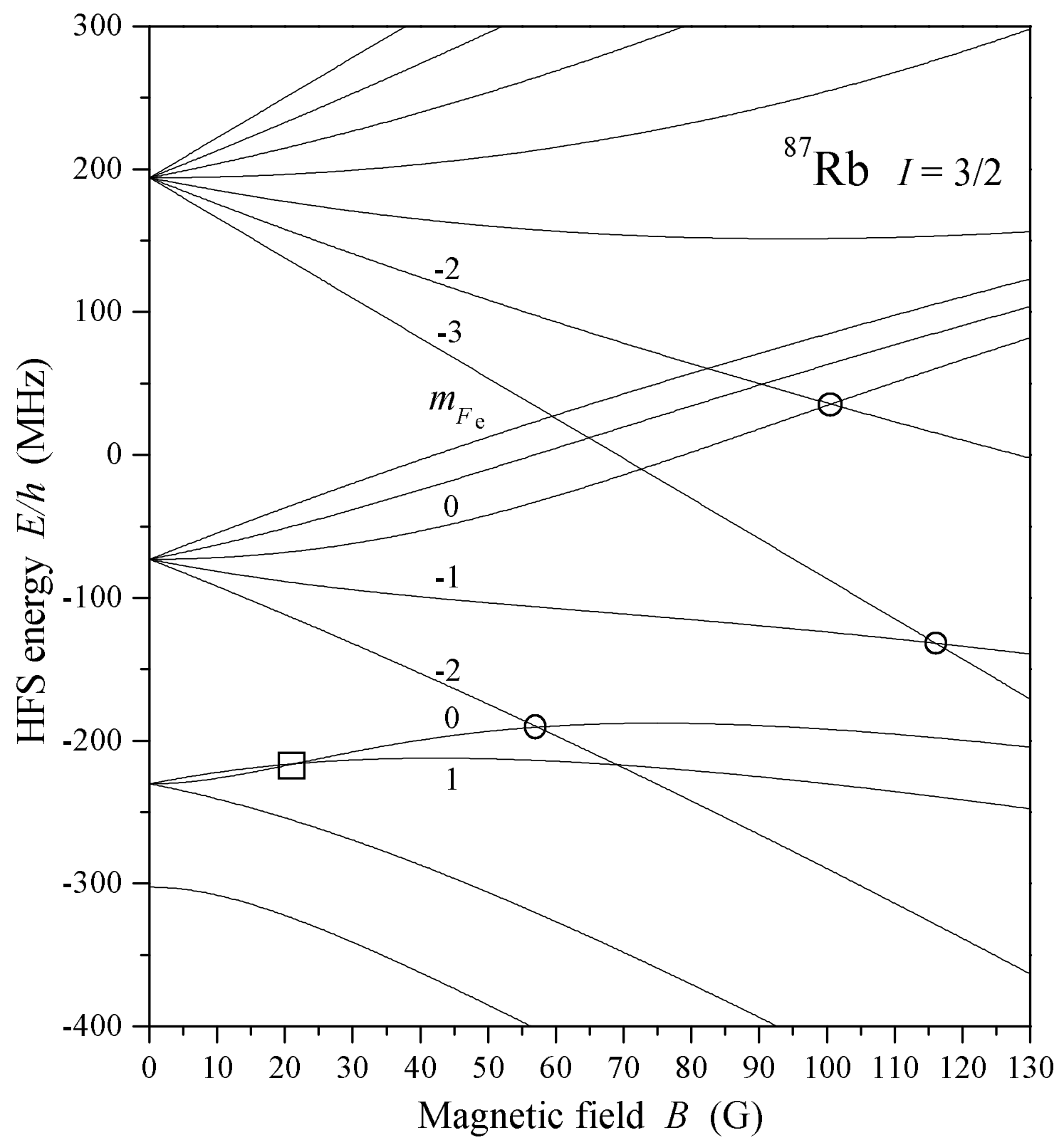

Figure 3

J.Alnis and M.Auzinsh 


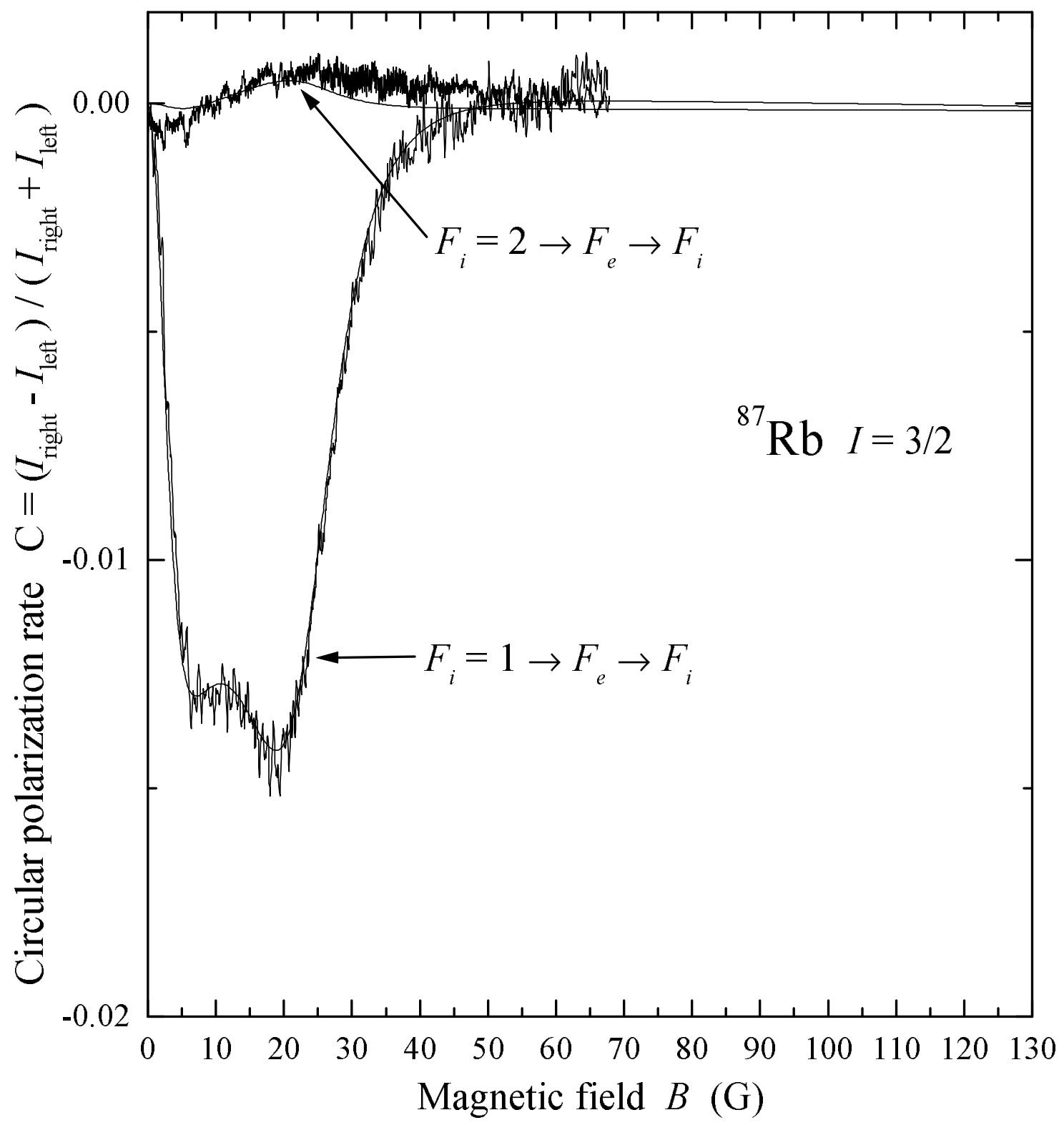

Figure 4

J.Alnis and M.Auzinsh 


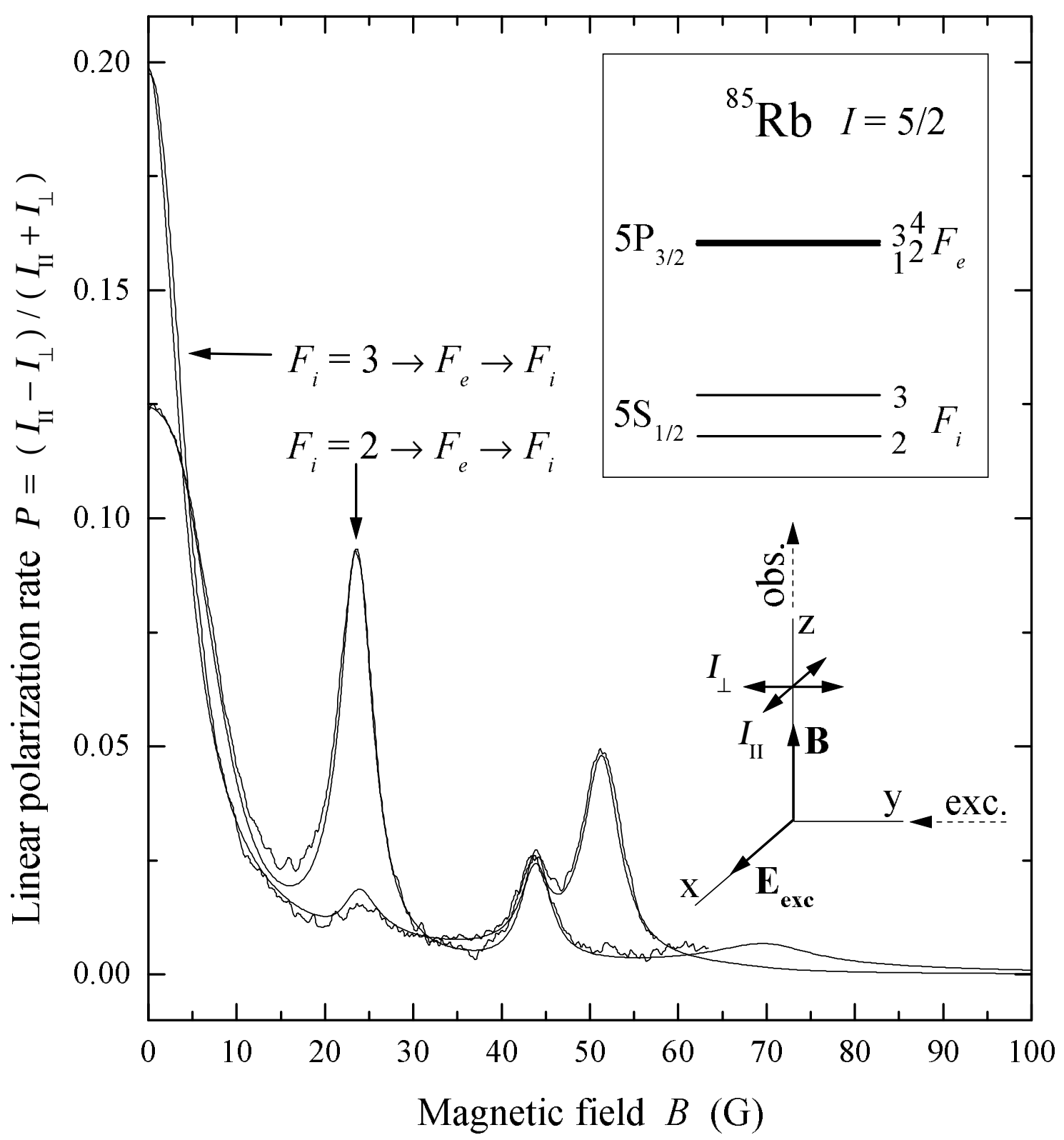

Figure 5

J.Alnis and M.Auzinsh 